\title{
PREVENCIÓN DEL DELITO Y FUTURO DE LA POLICÍA*
}

\section{Diego Torrente Robles}

Universidad de Barcelona

E-mail: torrente@riscd2.eco.ub.es

\begin{abstract}
RESUMEN
Este artículo trata de la prevención de la delincuencia y del futuro de la Policía. Parte de un análisis sobre cómo, a nivel de los países desarrollados, la preocupación social por una delincuencia creciente y el mito de una Policía antidelito ha puesto a ésta en una situación de encrucijada ante su propio futuro. El texto reproduce el debate que abrió David H. Bayley hace tres años y que todavía no está cerrado. La idea es que el futuro de la Policía depende de la respuesta a una pregunta. La de si está dispuesta a asumir la prevención de la delincuencia como una actividad central, y las consecuencias del cambio organizativo y cultural que ello comporta. El artículo discute las posibilidades de una respuesta negativa o afirmativa y las consecuencias que tendría para la Policía y la sociedad del futuro.
\end{abstract}

La Policía es una institución mítica. En general, el mundo cotidiano de la Policía es un mundo desconocido para los ciudadanos. Sin embargo, quizás no haya otra institución que se haya visto más retratada en la literatura, el cine y, en general, en los medios de comunicación. La imagen de la Policía en los medios de comunicación tiene una importancia central para reflejar la idea de

* Este artículo es una versión corregida y ampliada de una ponencia presentada en el Congreso Internacional sobre la Policía del Futuro: Mediación y proximidad, celebrado en L'Hospitalet de Llobregat (Barcelona) los días 13, 14 y 15 de noviembre de 1996. Fue debatida en la mesa redonda "Conflictos sociales y evolución de la demanda social a la policía».

\section{Reis}


una sociedad gobernada por el imperio de la ley ${ }^{1}$. Esa imagen es vital como apoyo a la autoridad policial y como elemento de legimitidad (Reiner, 1992a: 172). En la medida que una sociedad está más dividida y es más conflictiva y delictógena, esa imagen es más importante porque apoya a través de ella un consenso social en torno a la Policía.

Lo que más llama la atención de la imagen que reflejan la literatura, el cine y los medios de comunicación es que es inversa a la que reflejan las estadísticas y datos de la investigación científica sobre la realidad del delito y del trabajo policial $^{2}$. El delito aparece sobrerrepresentado en las formas más serias y violentas, en los casos esclarecidos, en la fase judicial, y con delincuentes algo más adultos. A su vez, el trabajo policial aparece reflejado en torno a la delincuencia y no en torno a las tareas de mantenimiento del orden cotidiano, que constituyen la mayoría de la actividad policial. Esa imagen irreal y poco exacta es útil a la Policía. Por una parte, esa visión de la delincuencia favorece una sensación de inquietud por la seguridad ciudadana y, por otra parte, una imagen de eficacia policial. Todo ello contribuye a crear una sensación de orden y justicia que legitima la actividad de la Policía y del sistema penal en general ${ }^{3}$.

Pero en los últimos años y en los países avanzados la Policía está siendo víctima de su propio mito social. Lo que se vuelve en contra de la Policía es la promesa, tan arraigada en su imagen pública, de contener la delincuencia aplicando la ley. Esta idea no sólo ha sido un mito externo, sino que también ha arraigado en la cultura profesional. La mayor parte del tiempo la Policía realiza funciones de mantenimiento cotidiano del orden y ofrece servicios a la población. Eso es algo que conocen bien los policías pero, paradójicamente, también la cultura interna ha contribuido a mantener ese mito. Muchos policías creen que el trabajo policial «real» consiste en aplicar la ley y frenar la delincuencia. Los otros trabajos son vistos a menudo como secundarios, sin apreciar del todo su importancia como factor de prevención de posibles situaciones de delincuencia.

Fruto de ese mito, la sociedad ha descargado en los hombros de la Policía, de forma principal, la tarea de la lucha contra la delincuencia. Pero la meta de acabar con, o siquiera reducir significativamente, la delincuencia escapa a las posibilidades reales de la Policía. Controlar los factores que la producen es algo

${ }^{1}$ En su libro Reporting Crime: The Media Politics of Criminal Justice, Phip Schlesinger y Howard Tumber (1994) subrayan cómo los medios de comunicación son el elemento más importante en la percepción ciudadana de la política y de las instituciones que realizan funciones políticas. Tanto es así que resulta difícil concebir la política sin los medios y los medios sin política.

Según David H. Bayley, los estudios norteamericanos muestran que sólo entre el 15 y el 20 por 100 de las llamadas tienen que ver con delincuencia; ese porcentaje está en torno al 17 por 100 en Inglaterra (Bayley, 1994: 17).

3 El mismo poder de los mass media en la legitimación social de la Policía lo tienen también en su deslegitimación a través de noticias sobre corrupción policial, ineficacia, abusos de autoridad, errores o escándalos. En todo caso, ello no hace sino confirmar la importancia de los medios para la Policía. 
que está fuera de su alcance. Por otra parte, la delincuencia comprende situaciones enormemente dispares. Comúnmente se piensa en la delincuencia común, en delitos sin víctima o en la oportunista, pero también existe la delincuencia de las organizaciones, de las profesiones, la delincuencia política, la profesional o el delito organizado, entre otras. La estructura de las organizaciones policiales hace más fácil afrontar un tipo de delincuencia más que otra. La Policía ha podido mantener el mito en la medida que la delincuencia se mantenía dentro de unos límites aceptables, mientras había dinero suficiente para compensar su crecimiento con ampliaciones de medios y plantillas, y mientras la seguridad no pasó a ser un tema social y político de primer orden. Pero en los años ochenta esos factores dejaron de darse y la Policía entró en una fase de cierta crisis de eficacia y de legitimidad.

En los últimos años se han producido en numerosos países desarrollados una serie de fenómenos que han hecho que este mito se haya vuelto en contra de la Policía. El primer factor de la crisis es el aumento de las tasas de delincuencia. En Inglaterra y Gales, por ejemplo, los años setenta elevan las estadísticas de delincuencia de forma importante al tiempo que decrecen las tasas de esclarecimiento. La cifra de un millón de delitos en 1964 se multiplica por dos en diez años, llegando a los cinco millones de delitos en 1990. En 1990 y 1991 la delincuencia crece interanualmente un 17 y 16 por 100 , respectivamente $^{4}$. El crecimiento de la delincuencia en España es todavía más acusado, a pesar de que las tasas de delitos por 100.000 habitantes están todavía alejadas de las inglesas y también su impacto político. En 1975 la Policía registra 132.476 delitos. Esa cifra se ve multiplicada casi por ocho en 1989, apreciándose 1.038.966 registros delictivos (el récord nacional). La década de los noventa ve un estancamiento y ligero decrecimiento en los delitos, pero no así en las faltas. El aumento de las desigualdades o fenómenos como la inmigración masiva y el delito organizado permiten aventurar la hipótesis que la fase de estancamiento quizás no dure mucho más. ¿Expresan esas cifras un crecimiento real? Las estadísticas policiales son problemáticas porque no recogen la denominada "cifra oscura" (es decir, la delincuencia real). Sus datos dependen básicamente de la denuncia ciudadana y del crecimiento del control policial. Sin embargo, los estudios de victimización muestran que existen razones para creer en un crecimiento, aunque más moderado, de la delincuencia «real».

Pero más importante que el crecimiento de la delincuencia es el hecho de que ésta se convierte en una cuestión social y política de primer orden. La población está preocupada y exige respuestas eficaces. Los partidos conservadores, que se eligen como defensores de la ley y el orden, abogan por una Policía más eficaz y un sistema punitivo más duro. Los partidos liberales y laboristas

${ }^{4}$ Si se toman las estadísticas de homicidio como indicador del problema de la delincuencia, según las estadísticas policiales, las tasas de homicidio por 100.000 habitantes en 1980 eran de 14,6 en Estados Unidos, 4 en Francia, 4,6 en Alemania, 2,9 en Bélgica, 1,2 en España y 1,6 en Inglaterra. 
coinciden en la demanda de mayor eficacia policial aunque desde una visión distinta. Se hacen eco de los estudios de victimización que muestran cómo la delincuencia perjudica especialmente a las capas más vulnerables y desprotegidas de la sociedad. El pensamiento progresista se aúna así al pensamiento conservador en su exigencia de reducción drástica e inmediata del delito. El delito se convierte así en una cuestión política de primer orden, y con él la Policía.

Todo ello coincide en un contexto de recesión económica. Por una parte, la idea de que a través del Welfare State es posible contener la delincuencia entra en crisis. Los datos muestran que la delincuencia no ha dejado de crecer. La prevención del delito a través de la política social pierde fuerza. Con ello la Policía concentra aún más las expectativas sociales de ser la barrera a la delincuencia. Por otra parte, diversos estudios muestran que invertir más dinero en Policía no lleva a una reducción en el número de delitos. En especial los gobiernos conservadores de Thatcher y Reagan pretenden reducir el tamaño del Estado. En Inglaterra, por ejemplo, existe un aumento del gasto policial en un primer momento con vistas a obtener logros claros. Sin embargo, esos logros no se dan y el énfasis se pone en obtener los mejores resultados posibles con el menor gasto. A comienzos de los noventa se introducen en la Policía criterios de gestión económica de forma que el presupuesto de cada Cuerpo depende de indicadores de funcionamiento.

En ese contexto se producen también algunos escándalos policiales y casos de violencia policial y corrupción que impactan en la opinión pública. Por otra parte, la crisis económica provoca un clima de convulsión social. La Policía se ve obligada a actuar con dureza. La huelga de los mineros ingleses es un caso paradigmático. Todo ello, junto con su cuestionada eficacia, hace que la legitimidad policial decrezca.

Tradicionalmente, los modelos de Policía racional burocráticos y profesionales han asumido la tarea de hacer cumplir la ley como el núcleo central de la labor policial y como método de lucha contra la delincuencia. La creencia en la eficacia de ese método empezó a tambalearse en los años setenta con la investigación empírica. El modelo profesional descansa en el patrullaje aleatorio, como técnica de detección y respuesta a la delincuencia, y en la investigación. Las investigaciones mostraron que, tanto para detectar el delito, como para iniciar diligencias, como para esclarecerlo, la Policía depende enormemente de la información que le facilita la población. Las distintas variantes de patrullaje tenían una incidencia mínima en cuanto a la eficacia de detección. Sólo el patrullaje a pie se demostró que tenía un efecto psicológico, que no de eficacia real, en la sensación de seguridad de la población. La eficacia esclarecedora de la investigación policial también se demostró que dependía en buena parte de la colaboración de la población.

La mejor forma de comprender el alcance de estas investigaciones es presentar los datos de una de ellas. Keith Bottomley y Clive Coleman publicaron en 1981 un estudio de una Policía inglesa. Las conclusiones de su trabajo son que la Policía depende en un 80 por 100 de los casos de la información que 
dan las víctimas para «descubrir» un delito. La Policía descubre el 13 por 100 y los testigos el 3 por 100 de los casos. Del 13 por 100 que detecta la Policía, la mitad lo hace interrogando a detenidos sobre acciones pasadas. Sólo un 6 por 100 de los delitos se descubren por acción directa de las patrullas. Según el British Crime Survey (la encuesta de victimización oficial británica), las víctimas sólo informan a la Policía del 41 por 100 de los delitos que experimentan y no al azar5. Del total de incidentes comunicados, y según el estudio anterior, en un 11 por 100 de casos el hecho no se reflejó las estadísticas. A la hora de esclarecer los delitos la acción policial aclara un 39 por 100 de los casos, las víctimas un 36 por 100, y el resto otros actores. Estos datos muestran cómo las víctimas, más que nadie, tienen gran influencia en definir el trabajo policial (Bottomley y Coleman, 1981: 53). Datos como éstos hicieron comprender fácilmente a la Policía la necesidad de mantener una buena sintonía con la población.

Como sostiene David H. Bayley, los modelos de organizaciones de Policía racional-burocráticos y profesionales están orientados a aplicar la ley, es decir, a procesar eficazmente los delitos que detectan, pero no están orientados a prevenir la delincuencia. Son organizaciones con orientación reactiva y no proactiva. Tratan con las consecuencias del delito pero no con sus causas. Este autor da varias razones de por qué la Policía no es eficaz en la lucha contra la delincuencia. La principal es que no consideran su misión prevenir. Los recursos no se utilizan racionalmente para prevenir. El patrullaje y la investigación, sus técnicas básicas, están pensados para responder a delitos que ya se han producido (la mayoría) o se están produciendo, pero no se anticipan a ellos. Para anticiparse a la delincuencia es necesario un cambio de orientación y organizativo radical. El trabajo es distinto, es necesario investigar y estudiar constantemente el entorno, detectar los problemas en contacto con la población. Pero también la organización del trabajo es distinta: es necesario dotar de gran autonomía a los equipos de barrio, acercar las decisiones a los problemas, que las iniciativas y políticas de actuación se diseñen de abajo arriba de la organización. Es necesario que las evaluaciones se hagan críticamente y con su mira puesta en la incidencia real en la delincuencia. Esos planteamientos suponen una revolución organizativa y filosófica (Bayley, 1994).

La Policía ha demostrado tener una capacidad autocrítica y de renovación como pocas organizaciones. El intento más serio de transformar a la Policía de reactiva en preventiva y aumentar la eficacia en la lucha contra el delito es lo que se conoce como policía comunitaria (community policing). Se desarrolla en los años ochenta como respuesta a la crisis policial. Existen visiones dispares de lo que quiere decir policía comunitaria y hasta dónde llevarla. Sin embargo,

Los robos en domicilio y de automóvil son más comunicados que los delitos de vandalismo o de robos personales. La British Crime Survey de 1982 muestra que las razones alegadas para no denunciar un delito a la Policía son: porque lo consideran un delito trivial (38 por 100), porque lo consideran un asunto inapropiado para la Policía y prefieren solucionarlo por sí mismos (33 por 100), porque creen que la Policía no puede hacer nada (16 por 100). 
todas ellas comparten una serie de rasgos. El primero es ver la necesidad de contar con la ayuda e implicación de los ciudadanos y la comunidad en la lucha contra el delito. El segundo, asumir inequívocamente que la Policía tiene como función principal la de prevenir la delincuencia. Prevenir es posible porque en el mundo delincuencial existen regularidades, pautas y víctimas comunes que es posible conocer y estudiar. El tercero es creer que es necesario un nuevo estilo de trabajo más proactivo, más estudioso, más informado, más verdaderamente profesional y menos reactivo. La policía comunitaria ha reinventado la Policía en cierto sentido.

¿Cómo puede prevenir la Policía? En realidad, ésa es una pregunta cuyas respuestas crecen día a día y con la experiencia. La delincuencia es un fenómeno multicausal del que sabemos poco en el fondo. La Policía está aprendiendo a prevenir a través de utilizar la imaginación en la búsqueda de soluciones y paliativos a los problemas y circunstancias que favorecen la delincuencia. Las dos filosofías más utilizadas: el problem solving y el situational crime prevention, se basan en trabajo caso a caso. Prevenir no es una cuestión de aplicar recetas. Las recetas hay que construirlas con arreglo a un método de cinco pasos: Primero, averiguando las necesidades de seguridad de la población. Las consultas más o menos regulares o institucionalizadas son la base para detectar los casos de delito. Segundo, diagnosticando las causas y analizando los contextos que producen delincuencia. Tercero, creando estrategias descentralizadas y especializadas. Es decir, adaptando las respuestas al lugar y al tipo de delito de que se trate. Cuarto, movilizando los recursos de la comunidad y los del Estado y coordinándolos. Y, finalmente, evaluando continuamente los resultados obtenidos. La prevención no significa dejar de aplicar la ley. Antes al contrario, aplicar la ley es una estrategia de prevención importante, pero junto a ella aparecen nuevos campos de posibilidades. El marco de la prevención es más grande y dentro de él cabe perfectamente el de hacer cumplir la legalidad.

¿Funciona la prevención? Las evaluaciones acerca del éxito de las organizaciones de policía comunitaria son poco concluyentes. Los modelos de policía comunitaria se han implantado, en general, de forma progresiva. La inercia organizativa racional-burocrática es grande y, por otra parte, la Policía no puede renunciar a su obligación de seguir aplicando la ley. En la mayoría de casos, la implantación queda limitada a grupos que operan en el marco de una organización tradicional. Sólo Japón, Singapur y algunos cuerpos aislados en Europa y Estados Unidos han asumido el modelo con integridad. En cualquier caso, los estudios realizados son más bien anecdóticos y no evaluaciones del impacto global en la delincuencia. Parece que es demasiado pronto para extraer conclusiones definitivas, máxime cuando la prevención se construye localmente y acumulando experiencia.

Más allá de si las experiencias de prevención policial hechas hasta ahora funcionan, la cuestión clave es si la Policía está dispuesta a asumir la prevención del delito como su función central. David Bayley dice que ésta es la cuestión más crítica que tiene que responder la Policía al encarar su futuro (Bayley, 
1994: 123). La decisión sitúa a las Policías en una encrucijada de la que no pueden escapar fácilmente. La pregunta es si las Policías deben (desde un punto de vista político, democrático y ético) y pueden (desde un punto de vista funcional y organizativo) asumir la responsabilidad de la prevención. Tanto la respuesta afirmativa como la negativa reportan consecuencias de largo alcance que se analizan.

El escenario imposible es el de eludir la cuestión y continuar como hasta ahora. Ciertamente, lo que se le exige a la Policía es que cumpla y haga cumplir la ley poniendo a disposición de los tribunales a los delincuentes. Sin embargo, lo que no es sostenible es que la Policía pueda afrontar una delincuencia creciente, ni pueda seguir sosteniendo promesas de frenarla, por ese método. Pero la responsabilidad no es sólo de la Policía, el mito se ha construido entre todos. Al final, insistir en esa línea lleva al sistema penal a endurecer las condenas, a soportar la tensión de una superpoblación carcelaria que es a todas luces inviable y a una mayor violencia policial, al trabajar ésta bajo una gran presión por la eficacia y ante una deslegitimidad creciente. La Policía ha obtenido muchos recursos económicos en base a esa promesa. Sin embargo, esa situación ya no es sostenible por más tiempo debido a los factores señalados.

Una alternativa es rechazar de plano la responsabilidad de la Policía en la prevención y reconocer honestamente que no puede frenar el delito. El argumento podría ser que la Policía no puede lograrlo porque el objetivo está fuera de su alcance y las causas delictivas fuera de su control. La alternativa es seguir detectando y procesando el delito que buenamente se pueda. No en vano ésta es su función tradicional y algo que hay que seguir haciendo porque es un servicio valioso a la sociedad. Nadie se atrevería a quitar el teléfono policial donde se reciben las llamadas de urgencia de la población. Ese servicio es básico. Sin embargo, ésta es una postura arriesgada políticamente debido a que significaría defraudar expectativas y reconocer que se engañó previamente respecto a la eficacia policial.

Sin embargo, la cuestión más importante desde el punto de vista de la sociedad no es ésa. Lo verdaderamente crítico es que la delincuencia seguiría creciendo y también la preocupación pública. Alguien debería asumir la responsabilidad de combatirla y prevenirla, pero quién. ¿Cómo organizar una institución nueva? Hasta ahora la sociedad no tiene una institución específica para ello. Hasta finales de los setenta se pensó que el gasto social cumplía una función preventiva frente a la delincuencia al paliar la desigualdad y la pobreza. Más tarde se impone la visión, y en especial ante la realidad de la crisis económica, de que éste es un sistema muy caro y de eficacia limitada ${ }^{6}$. Hoy en día, en un momento de dificultades fiscales, la idea de la prevención a través del Estado del Bienestar parece haber perdido cierta fuerza. Al abandonar esta línea, los políticos han descargado en la Policía todo el peso de la responsabili-

${ }^{6}$ Ésta es una posición que rebate Steven Box en su libro Recession, Crime and Punishment, donde se reevalúan esos estudios. 
dad por la delincuencia. La posibilidad de «inventar» una institución nueva (con sus expertos, infraestructura y conocimientos) que se ocupe de prevenir parece bastante inviable. Algo así no se improvisa de repente.

La otra alternativa de futuro es que la Policía asuma decididamente y sin ambivalencias la prevención como su objetivo central. Según Sir Robert Peel, el fundador de la Metropolitan Police de Londres, ésta es la función básica de la Policía. Si se acepta que alguien tiene que asumir la responsabilidad por prevenir la delincuencia, la idea podría no ser tan descabellada: la Policía cuenta con infraestructuras organizativas, experiencia, conocimientos de la ciudad y de la delincuencia. Resultaría más barato, probablemente más efectivo y más realista reconvertir las organizaciones policiales existentes que crear una institución nueva para la prevención. Éste sería un proceso largo y construido desde la base. No se trata de que la Policía haga todo. No es únicamente a la Policía a quien le corresponde solucionar la delincuencia, sino al resto de la sociedad. En el nuevo sistema la Policía asumiría el rol de grupo experto que desde una base empírica formula propuestas al resto de la sociedad. La idea es que, a partir del análisis de caso por caso y día a día, se vayan encontrando regularidades que ayuden a entender mejor la delincuencia y a formular estrategias. El reto de la Policía comunitaria o preventiva consiste en seguir aplicando la ley, no desalentar otras iniciativas de prevención y no dar exceso de poder a la Policía.

Una apuesta decidida por la prevención supone un cambio drástico en el rol de la Policía. Un cambio que tiene consecuencias importantes para las sociedades democráticas. La prevención lleva a que la Policía asuma más responsabilidad y un rol nuevo y más profesional. La investigación limitada al delincuente y al delito se amplía; ahora es la comunidad en general el objeto de la indagación policial ${ }^{7}$. Por otra parte, a su papel como agente en el sistema de justicia penal se le suma otro nuevo respecto al Poder Ejecutivo. La Policía pasa a ser un actor con mayor influencia política en tanto que, como grupo experto, propone al Ejecutivo iniciativas de actuación coordinada contra la delincuencia de diversas instituciones. La idea de que la Policía asuma ese liderazgo social supone una idea sin precedentes y que rompe los moldes actuales.

Entrar de lleno en un modelo policial preventivo conlleva una paradoja. En los años sesenta los sociólgos descubrieron que la Policía opera con márgenes amplios de discrecionalidad. Ello tuvo un fuerte impacto social y provocó el auge de los modelos racional-burocráticos, el modelo que ahora se quiere superar, en un intento de sujetar la Policía a la ley. Ahora, el modelo de Policía preventiva puede representar pasar de una Policía de «mínimos», reactiva y sujeta a la ley, a otra de máximos, proactiva y con márgenes amplios de autonomía y poder. Una Policía con estos nuevos roles crea un dilema a las sociedades

Stanley Cohen comenta refiriéndose al Situational Crime Prevention cómo los sistemas de control social modernos tienden a ir de unos controles centrados en la figura del delincuente a unos controles focalizados en el conjunto de la población (Cohen, 1985). 
democráticas. El problema más importante que se plantea es el de la regulación de su poder y el diseño de nuevos mecanismos de control. El nuevo marco tiene que fijar un equilibrio difícil entre garantizar el desarrollo de la imaginación y la creatividad orientada a la prevención, y la protección de los derechos y libertades del ciudadano.

\section{BIBLIOGRAFÍA}

Bayley, David H. (1994): Police for the Future (Nueva York: Oxford University Press), 187 pp. Bottomley, A. Keith, y Coleman, Clive A. (1981): Understanding crime rates: Police and Public Roles in the Production of Criminal Statistics (Fornborough: Gower), $170 \mathrm{pp.}$

Box, Steven (1987): Recession, Crime and Punishment (Londres: McMillan Education), 244 pp.

Christie, Nill (1993): Crime Control As Industry (Oslo: Scandinavian University Press, y Londres: Routledge), 192 pp.

Clarke, Ronald V. G. (ed.) (1992): Situational Crime Prevention: Successful Case Studies (Albany, Nueva York: Harrow \& Heston), 286 pp.

CoHen, Stanley (1988): Visiones de control social (Bardcelona: PPU), $407 \mathrm{pp}$.

Downes, David, y Rock, Paul (1995): Understanding Deviance: A Guide to the Sociology of Crime and Rule Breaking (Nueva York: Clarendon Press), 415 pp.

Fennell, Phil, et al. (eds.) (1994): Criminal Justice in Europe: A Comparative Study (Oxford: Clarendon Press), 404 pp.

Johnston, Les (1991): The Rebirth of Private Policing (Londres: Routledge), $251 \mathrm{pp.}$

Lustgarten, Laurence: The Governance of Police (Londres: Sweet \& Maxwell), 197 pp.

Marguire, Mike (1994): "Crime statistics and their interpretarions», The Oxford Handbook of Criminology (Cambridge: Oxford), 1258 pp.

MARTín, Manuel (1990): La profesión de policía (Madrid: Centro de Investigaciones Sociológicas y Siglo XXI).

- (1992): «Policía, profesión y organización: hacia un modelo integral de la Policía en España", REIS, 59 (julio-septiembre): 205-222.

Mayhew, Pat; Maung, Natalie, y Mirrlees-Black (1993): Catriona, The 1992 British Crime Survey (Londres: Home Office), 195 pp.

Morris, Pauline, y Heal, Kevin: Crime Control and the Police (Londres: HMSO, 1989), 67 pp. Home Office Research Study 67.

Newman, Oscar (1972): Defensible Space: Crime Prevention Trhough Urban Design (Nueva York: MacMillan), 264 pp.

ReIner, Robert (1992a): The Politics of the Police (Brighton: Wheatsheaf), 334 pp.

- (1992b): «Policing A Postmodern Society», Modern Law Review, vol. 55, n.o 6, pp. 761-781.

SChlesinger, Philip, y Tumber, Howard (1994): Reporting Crime: The Media politics of Criminal Justice (Oxford: Clarendon Press; Nueva York: Oxford University Press), 287 pp .

SKOLNICK, Jerome H. (1975): Justice Without Trial: Law Enforcement in Democratic Society (Nueva York: Willey), 309 pp.

Torrente, Diego (1977a): «Hacer la ronda: Análisis sociológico de las actuaciones de la Policía municipal», Revista Internacional de Sociología, 16: 139-179.

- (1977b): La sociedad policial: poder, trabajo y cultura en una organización local de Policía (Madrid: Centro de Investigaciones Sociológicas-Universidad de Barcelona), 261 pp.

- (1993): «Investigar la policía», REIS, 59.

Wilson, James Q. (1983): Thinking about Crime (Nueva York: Basic Books), 293 pp. 


\begin{abstract}
This paper is about crime prevention and the future of police. It analyzes how, in developed countries there is an increasing social concern about the growing crime rates. Also there is a myth of Police as a crime fighting agency. This two facts are placed the future of the police in a difficult situation. This presentation echoes David Bayley's debate. He holds that the future of the Police depends basically on the answer to a key question. This question is whether Police is going to take full responsibility in crime prevention. This reponsibility will lead to major organizational and cultural changes in the Police. This paper discuss the possibilities about a positive or negative answer and the consequences that it would have for the Police and the society.
\end{abstract}


NOTAS DE INVESTIGACIÓN 